\title{
Der Gelenkinfekt ohne Implantat
}

Marc Hückstädt, Gunter O. Hofmann

\section{Einleitung}

\section{Definition}

\section{Merke}

Eine Gelenkinfektion ist definiert als der Befall eines Gelenks durch pathogene Erreger, meistens Bakterien, und eine sich anschließende Entzündung [6].

\section{Epidemiologie}

Die Häufigkeit nicht implantatassoziierter Gelenkinfektionen wird mit bis zu 8/100000 Einwohner pro Jahr angegeben. Die Inzidenz ist aufgrund der demografischen Entwicklung, der zunehmenden Zahl arthroskopischer und offen-chirurgischer Eingriffe sowie diagnostischer und therapeutischer Punktionen von Gelenken und dem zunehmenden Einsatz immunsuppressiver Medikamente sowohl in Europa als auch den USA steigend [5].

\section{Ätiologie und Pathogenese}

Prinzipiell können endogene und exogene Ursachen einer Gelenkinfektion unterschieden werden [6]. Im erstgenannten Fall kommt es im Rahmen einer Bakteriämie oder Sepsis zur hämatogenen Infektion eines Gelenks mit pathogenen Erregern. Bei einer exogenen Infektion führen operative Eingriffe, perforierende Gelenkverletzungen oder Verletzungen bzw. Infektionen in Gelenknähe (Bursitis) zur Kontamination und folgenden Infektion eines Gelenks. Prinzipiell kann in allen Gelenken eine Infektion auftreten, wobei das Kniegelenk, gefolgt von Schulter- und Ellenbogengelenk am häufigsten betroffen ist.

Risikofaktoren für endogene Gelenkinfektionen [1, 6]:

- rheumatoide Arthritis (Inzidenz der septischen Arthritis: $180 / 100000$ [4])

- weitere Erkrankungen des rheumatischen Formenkreises mit Gelenkbeteiligung

- Menstruation, Schwangerschaft

- Zeckenbiss (Borreliose)

- Systemerkrankungen mit Immunschwäche

- immunsuppressive Medikamente (Disease modifying antirheumatic Drugs = DMARD), vor allem TNF- $\alpha$-Blocker

- intravaskuläre Katheter, Urinkatheter

- Sepsis

- i.v. Drogenabhängigkeit
- Diabetes mellitus in Abhängigkeit von Typ, Einstellung und Erkrankungsdauer

- Alter <2 Jahre, Alter $>80$ Jahre

- degenerative Gelenkerkrankungen (Arthrose), Gicht, Chondrokalzinose

Risikofaktoren für exogene Gelenkinfektion [1,6]:

- gelenknahe Wunden, insbesondere mit primärer Gelenkverletzung

- gelenknahe Hautinfektionen (Impetigo contagiosa, Erysipel, Pusteln)

- gelenknahe Bursitiden

- offene Gelenkfrakturen und gelenknahe offene Frakturen

- Senkungsabszesse

- intra- und periartikuläre Injektionen (Inzidenz bis 0,3\%)

- diagnostische und therapeutische Arthroskopie (Inzidenz bis $0,5 \%$ )

- offene Operation an Gelenken (Inzidenz bis über 1\%)

- Operationen in Gelenknähe

- postoperatives Hämarthros

- degenerative Gelenkerkrankungen (Arthrose), Gicht, Chondrokalzinose

\section{Einteilung}

Die Klassifikation einer Gelenkinfektion kann nach verschiedenen Kriterien erfolgen, einige wichtige Einteilungen seien im Folgenden aufgeführt. Die Klassifikation mit Stadieneinteilung ist klinisch bedeutsam, da sich unmittelbare therapeutische Konsequenzen ergeben. Die Übergänge zwischen den Stadien sind dabei fließend.

1. Klassifikation nach dem Zeitraum ab dem Symptombeginn

- Frühinfekt: Dauer der Symptome weniger als 6 Wochen

- Spätinfekt: Symptomdauer über 6 Wochen

2. Klassifikation nach klinisch-morphologischen Veränderungen (Kuner 1987, [1,7])

- Stadium I (purulente Synovialitis): Gelenkschwellung, überwärmte, gerötete und glänzende Haut, Ergussbildung, Schonhaltung

- Stadium II (Gelenkempyem): zusätzlich periartikuläre Schwellung und Rötung, starke Schmerzhaftigkeit, Druckschmerz über der Kapsel, Entlastungsstellung in Beugung, ggf. Fieber

- Stadium III (Panarthritis): massive Weichteilschwellung, prall gespannte, glänzende Haut, extreme 


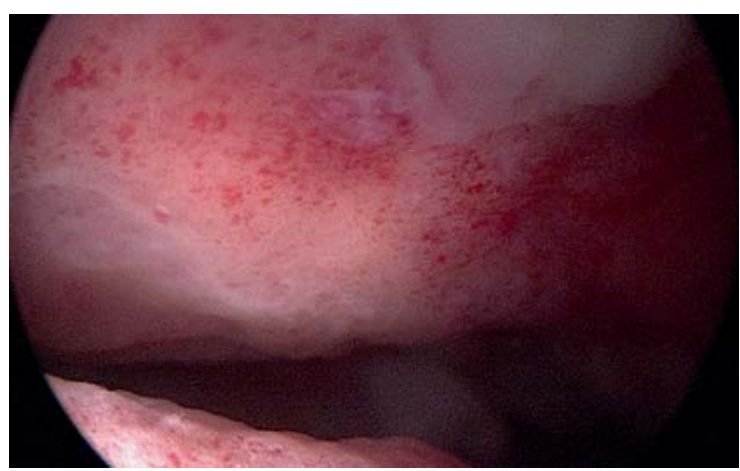

- Abb. 1 Akute septische Arthritis im Stadium Gächter I, das Bild zeigt eine Rötung der Synovia mit petechialen Einblutungen.

Schmerzhaftigkeit, septische Temperaturen, Beeinträchtigung des Allgemeinzustands

- Stadium IV (chronische Arthritis): geringe Entzündungszeichen, Deformierung und diffuse Schwellung des Gelenks, Fistelbildung oder starke Vernarbung, schmerzhafte Instabilität, starke funktionelle Behinderung

3. Klassifikation nach dem arthroskopischen Befund (A. Gächter, 1994, [3, 5])

- Stadium I: leicht trüber Erguss, Gelenkschleimhaut gerötet, evtl. petechiale Blutungen

- Stadium II: ausgeprägte Synovitis, Fibrinausschwitzungen, eitriger Erguss

- Stadium III: Zottenbildung, Kammerung, Ausbildung eines sog. „Badeschwamms“

- Stadium IV: Synovialmembran wächst infiltrierend in den Knorpel und unterminiert ihn, radiologisch Arrosionen, subchondrale Aufhellungen, Zysten

Die $\mathbf{A b b}$. 1-3 zeigen verschiedene arthroskopische Stadien einer bakteriellen Kniegelenkinfektion.

\section{Diagnostik}

\begin{abstract}
Merke
Nur in seltenen Fällen sind einzelne diagnostische Kriterien (direkter Erregernachweis in der Gram-Färbung eines Gelenkpunktats) beweisend für das Vorliegen einer septischen Arthritis, sodass eine Vielzahl diagnostischer Kriterien für die Differenzialdiagnostik einer Arthritis bedeutsam ist. Zu beachten ist, dass eine laufende antibiotische Therapie die Symptome einer Gelenkinfektion abschwächen und somit maskieren kann.
\end{abstract}

\section{Anamnese}

Trotz der breiten Verfügbarkeit geeigneter bildgebender Verfahren und moderner Laboruntersuchungen stellt die Anamnese auch heute noch einen wesentlichen Bestandteil der Diagnostik von Gelenkinfektionen dar. Im Folgenden werden wichtige Kriterien der Akutanamnese und der allgemeinen Anamnese dargestellt [1]

\section{Akutanamnese:}

- vorausgegangene Unfälle und Erkrankungen

- Verletzungen in Gelenknähe ( $\bullet$ Abb. 4)

- vorausgegangene Maßnahmen (Gelenkpunktion, Operation)

- vorausgegangene systemische oder lokale gelenknahe Infektionen

- Dauer der Symptome

- weitere Symptome abgesehen von den Gelenkbeschwerden (Diarrhö, Fieber, Allgemeinzustand, Zeckenbiss, Urethritis)

- Sexualanamnese

- Erythema nodosum

- vorangegangene oder laufende antibiotische Therapie (larvierte Infektion!)
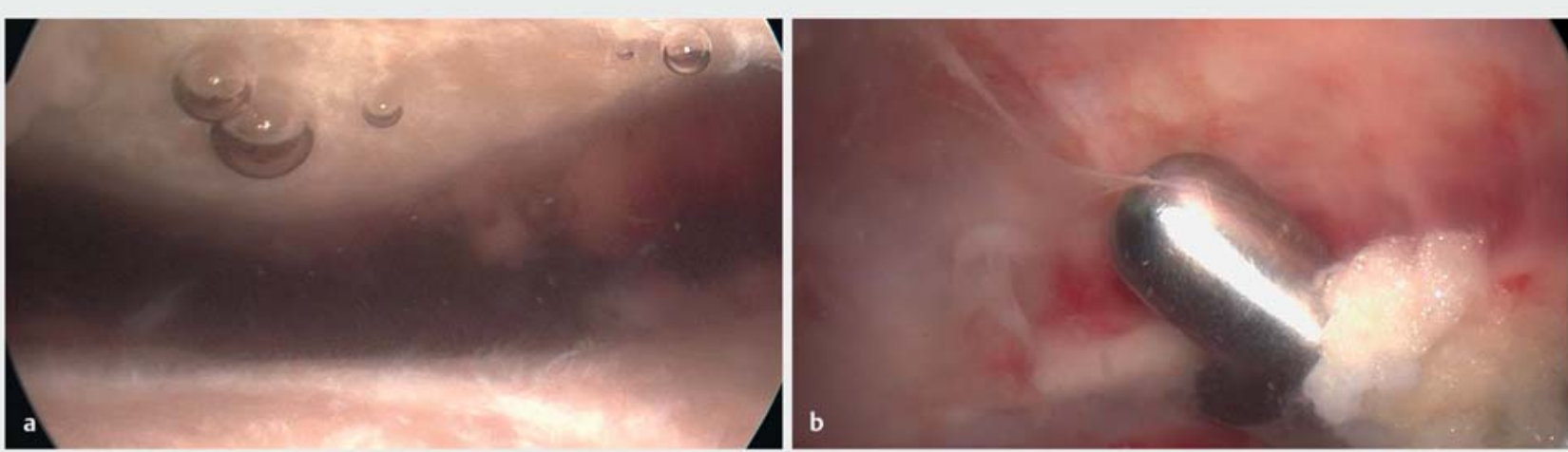

- Abb. 2 Eitriger Gelenkerguss (oben), eine Rötung der Synovia und Fibrinablagerungen (unten links) als Zeichen einer septischen Arthritis im Stadium II nach Gächter. 


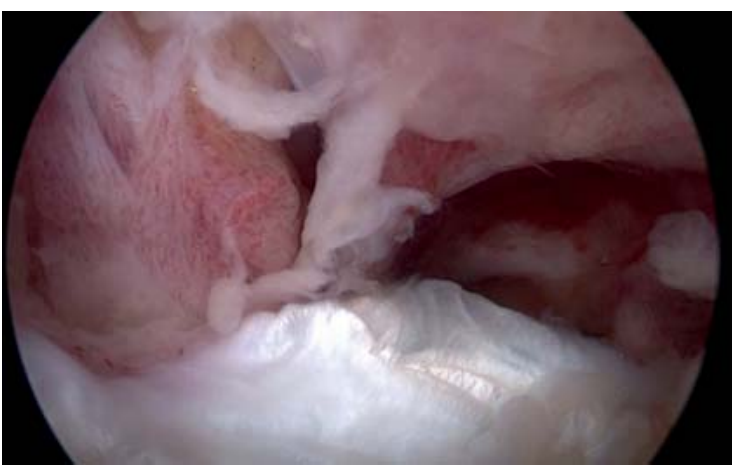

- Abb. 3 Chronifizierte septische Arthritis des Kniegelenks, Stadium III nach Gächter, das arthroskopische Bild zeigt neben einer Rötung der Gelenkschleimhaut eine Verdickung der Synovialmembran und beginnende Kompartmentformationen („Badeschwamm“).

Allgemeine Anamnese:

- Medikamente (DMARD), Nikotin, Alkohol, Drogen

- Vorerkrankungen

- chronische Infektionen (Hepatitis B und C, HIV)

- Stoffwechselerkrankungen (Diabetes mellitus, Gicht)

- Erkrankungen des rheumatischen Formenkreises mit Gelenkbeteiligung (frühere Arthritiden)

- Gefäßerkrankungen (arterielle Verschlusskrankheit, postthrombotisches Syndrom)

- degenerative Gelenkerkrankungen (Arthrose)

- maligne Erkrankungen

- Psoriasis

\section{Klinische Untersuchung}

Ähnlich wie der Anamnese kommt der klinischen Untersuchung eine herausragende Bedeutung in der Diagnostik von Gelenkinfektionen zu. Neben der lokalen klinischen Untersuchung des betroffenen Gelenks können weitere klinische Befunde für die Diagnosestellung wegweisend sein:

- „klassische Entzündungszeichen“: Rötung, Schwellung, Schmerz, Überwärmung, Funktionsverlust (Schonhaltung des Gelenks)

- Gelenkerguss („tanzende Patella“)

- Wunden, Narben, Fisteln

- Fieber

- reduzierter Allgemeinzustand

- weitere Sepsiszeichen

- weiterer lokaler Infektfokus

- Dauerkatheter, i.v. Zugänge

\section{Laboruntersuchungen}

Neben Anamnese und klinischer Untersuchung stellen Laboruntersuchungen einen weiteren wichtigen Baustein in Diagnostik und Differenzialdiagnostik der Arthritiden dar. Oft sind die Befunde wegweisend zur Diskriminierung akuter bakterieller Infektionen von rheumatischen

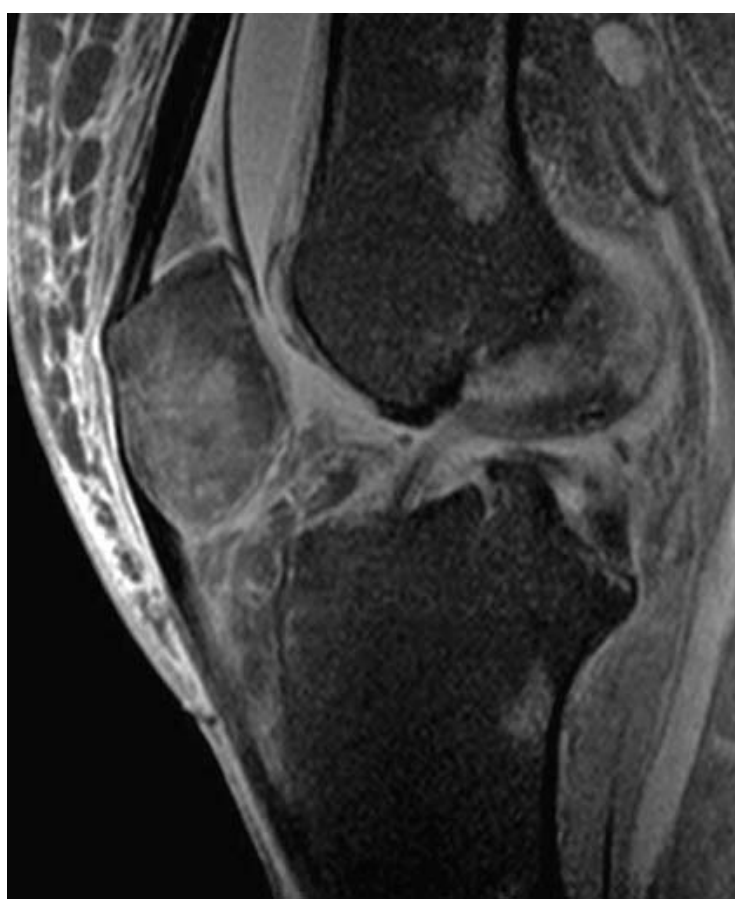

- Abb. 4 Chronische bakterielle Gonarthritis im Stadium Gächter IV nach Durchspießungsverletzung des Kniegelenks mit einem verschmutzten Draht, 38-jährige Patientin ohne weitere Risikofaktoren, verzögerter Behandlungsbeginn ohne primäre Revision des Kniegelenks! Die MRT zeigt neben einem Gelenkerguss und einer chronischen Synovialitis eine begleitende Osteitis der Patella.

Erkrankungen oder parainfektiösen Arthritiden. Folgende Laboruntersuchungen werden in unserer Klinik bei Verdacht auf eine septische Arthritis standardmäßig durchgeführt:

- kleines Blutbild, Gerinnungsparameter

- C-reaktives Protein (CRP), Procalcitonin (PCT)

- Kreatinin, Elektrolyte

- Harnsäure

- Blutkultur (bei Fieber oder weiteren Sepsissymptomen)

- weitere Laboruntersuchungen unter Berücksichtigung von Alter und Begleiterkrankungen der Patienten

Bei unklaren Befunden (geringe bis mäßige Erhöhung der Entzündungswerte, fehlende Risikofaktoren für exogene oder endogene Gelenkinfektionen, lange Krankheitsverläufe, junge sexuell aktive Erwachsene, Hinweise auf Erkrankungen des rheumatischen Formenkreises oder auf parainfektiöse Arthritiden, Psoriasis, Sakroiliitis) sollte die Labordiagnostik diesbezüglich erweitert werden:

- antinukleäre Antikörper (ANA), extrahierbare nukleäre Antigene (ENA)

- Antistreptolysin (ASL), Citrullin-Antikörper (CCP)

- humanes Leukozytenantigen B27 (HLA-B27)

- serologische Diagnostik der parainfektiösen Arthritiden 


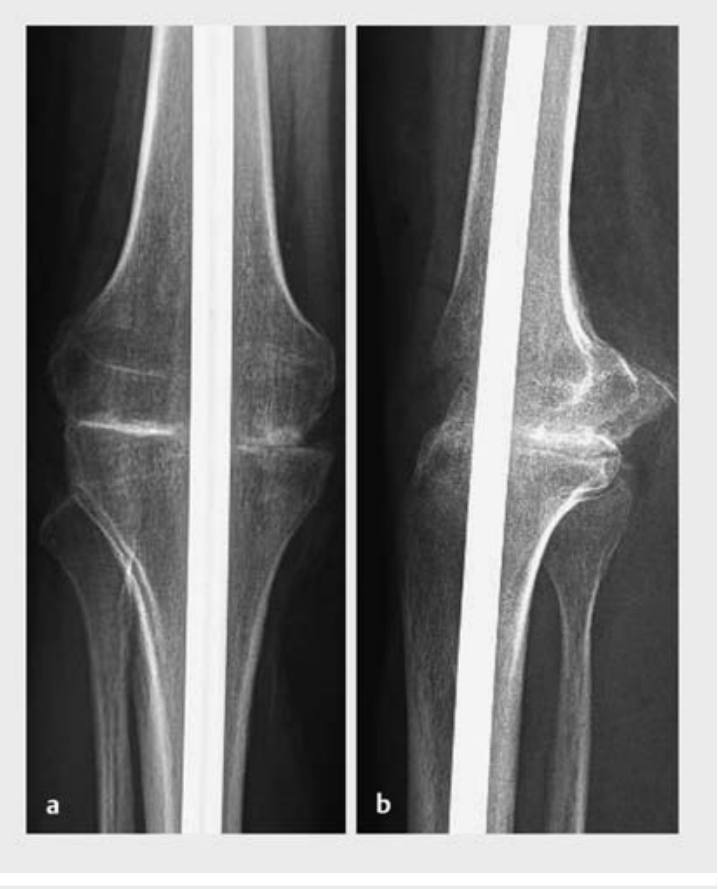

- Abb. 5 Trotz mehrfacher arthroskopischer und offenchirurgischer Sanierungsversuche sowie adäquater antibiotischer Therapie kam es zu mehrfachen Rezidivinfektionen. Die Sanierung gelang schließlich durch eine Gelenkresektion mit Resektion der Patella und eine folgende Arthrodese des Kniegelenks. Auch im weiteren Verlauf traten keine Zeichen eines Rezidivs auf, die Patientin ist nahezu beschwerdefrei, sie ist stabil und gut adaptiert mobilisiert und arbeitsfähig bei vollständigem Verlust der Mobilität des Kniegelenks.
- Chlamydia trachomatis, Lues (besonders junge, sexuell aktive Erwachsene)

- Borrelien (die Zeckenbissanamnese kann leer sein!)

- Yersinien, Salmonellen, Shigellen, Campylobacter

- eine Vielzahl weiterer bakterieller und viraler Erreger können parainfektiöse Arthritiden verursachen

Bei negativer Labordiagnostik und anhaltenden Beschwerden muss die Diagnostik ggf. in Absprache mit einem Rheumatologen erweitert werden.

\section{Bildgebende Diagnostik}

Bildgebende Verfahren gehören zur Standarddiagnostik der Arthritis. Auch wenn sich die Genese einer Arthritis mit den verfügbaren Methoden meist nicht sicher klären lässt, können aus den Untersuchungen wichtige diagnostische und therapeutische Schlüsse gezogen werden.

- Röntgenuntersuchung: Standardverfahren, Hinweise auf traumatische oder degenerative Gelenkveränderungen, Knochenbeteiligung, Gelenkerguss

- Sonografie: Standardverfahren, Nachweis eines Gelenkergusses oder eines Hämarthros, Kapselverdickung, begleitende Pathologie der Weichteile (Abszess), Möglichkeit der sonografisch gestützten Punktion eines Gelenks

- Computertomografie (CT): nur bei spezieller Fragestellung, genaue Beurteilung knöcherner Strukturen ( $\triangleright$ Abb. 6), Alternativverfahren (mit Kontrastmittel) bei Nichtdurchführbarkeit einer Magnetresonanztomografie (MRT)

- MRT: hochsensitives Verfahren zur Erfassung entzündlicher Veränderungen praktisch aller Gelenke, degenerativer und traumatischer Veränderungen, einer knöchernen Mitbeteiligung und von Begleitpathologien (Spondylodiszitis usw., - Abb. 4)
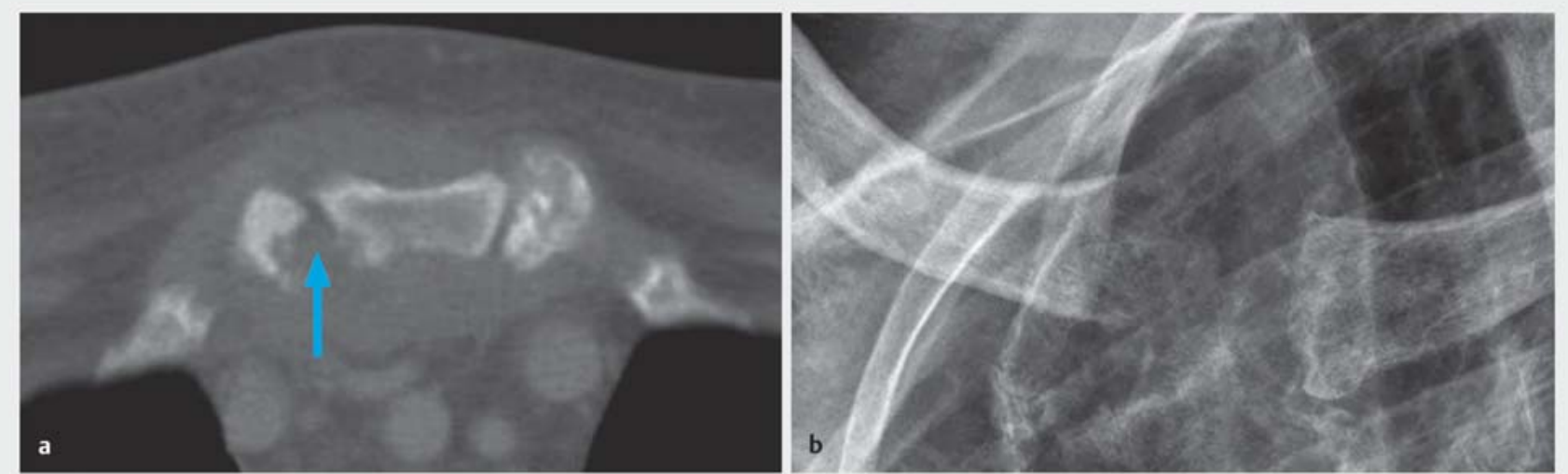

- Abb. 6 Die CT (linkes Bild) zeigt eine bakterielle Arthritis (Staphylococcus aureus) auf dem Boden einer rheumatoiden Arthritis mit ausgeprägter Zerstörung vor allem des rechten Sternoklavikulargelenks (Pfeil) und umgebenden Abszess. Bei schwerem septischem Verlauf gelang die Sanierung der Infektion bei der 73-jährigen Patientin durch eine Resektion beider Sternoklavikulargelenke (Resektionsarthroplastik) und des Manubrium sterni bei begleitender Osteitis (rechtes Bild) sowie durch eine gezielte antibiotische Therapie. 
- Fisteldarstellung mit Kontrastmittel: seltene Indikation bei Vorliegen einer Fistel und unsicherer Gelenkbeteiligung

- 3-Phasen-Skelettszintigrafie: seltene Indikation bei unklaren Befunden

\section{Gelenkpunktatuntersuchungen}

Die Leitlinienkommission der Deutschen Gesellschaft für Unfallchirurgie (DGU) gibt bez. der Gelenkpunktion folgende Empfehlung [1]:

Merke

„Die Punktion des betroffenen Gelenkes erfolgt i. d. R. präoperativ, bei klarer Operationsindikation ist sie jedoch nicht erforderlich. Die mikrobiologische Untersuchung von Flüssigkeiten und Gewebsanteilen ist der Abstrichuntersuchung vorzuziehen.“ Gelenkpunktionen müssen unter strengster Beachtung der Asepsis durchgeführt werden!

Die Qualität des Punktats (eitrig, blutig, serös) soll dokumentiert, das Punktat selbst in geeigneten sterilen Behältnissen zur mikroskopischen, mikrobiologischen und ggf. histologischen Untersuchung eingesandt werden. Das Untersuchungsmaterial soll umgehend ins Labor transportiert und verarbeitet werden.

\section{Mikrobiologische Untersuchungen}

Der mikrobiologische Nachweis der verursachenden, meist bakteriellen Erreger inklusive der Resistenzbestimmung ist für eine optimale Therapie unerlässlich. Aus diesem Grund nimmt dieses diagnostische Kriterium einen besonderen Stellenwert ein. Mehrere Möglichkeiten mikrobiologischer Diagnostik stehen zur Verfügung und sollten, wenn möglich, genutzt werden. Eine fachgerechte Durchführung ist dabei wichtig, da in bis zu $30 \%$ mit falsch negativen Befunden zu rechnen ist [8]. Wenn irgend möglich, sollten die mikrobiologischen Proben vor Beginn der kalkulierten antibiotischen Therapie gewonnen werden.

- mikroskopische Direktuntersuchung des Gelenkpunktats: Leukozytenzählung (Leukozytenwerte zwischen 1000 und 10000 Leukozyten/mm³ schließen eine bakterielle Infektion nicht aus [1,2])

- Gram-Präparat des Gelenkpunktats: hochspezifisch, schnelles Ergebnis, unmittelbare therapeutische Konsequenz

- Laktatspiegel des Gelenkpunktats: Erhöhung des Laktats ist Zeichen einer bakteriellen Infektion (anaerobe Glykolyse der Bakterien)

- Kulturuntersuchung des Gelenkpunktats mit Resistenzbestimmung

- Kulturuntersuchung operativ gewonnener Gewebeproben (Synovialis) mit Resistenzbestimmung (sensitiver als Kultur aus einem Abstrich)

- Blutkultur: bei Fieber und weiteren Sepsissymptomen, Wiederholung der Untersuchung ist zu empfehlen
- Bestimmung von $\alpha$-Defensin: Defensine sind körpereigene antimikrobielle Substanzen, die in infizierten Gelenkflüssigkeiten in hoher Konzentration nachgewiesen werden können. Entsprechende Tests sind kommerziell verfügbar. Ob die Bestimmung von $\alpha$-Defensin der klassischen Synoviaanalyse überlegen ist, müssen weitere Untersuchungen zukünftig unter Beweis stellen.

Prinzipiell kann eine Vielzahl bakterieller Erreger eine septische Arthritis verursachen. Pilzinfektionen von Gelenken sind dagegen selten und meist Zeichen einer Immunsuppression oder einer lang andauernden antibiotischen Vorbehandlung. Die häufigsten bakteriellen Erreger seien im Folgenden genannt, Konsequenzen im Hinblick auf eine kalkulierte antibiotische Therapie lassen sich aus der Häufigkeitsverteilung ableiten [1,6]. Mischinfektionen sind möglich und treten vor allem bei lang andauernder Behandlung und offenen Gelenkverletzungen auf.

- Staphylococcus aureus: bis $80 \%$

- Streptokokken (Gruppen A und B): bis 20\%

- gramnegative Erreger: bis 16\%

- Pneumokokken: bis 5\%

- Gonokokken

- Haemophilus influenzae Typ B (rückläufig durch Impfung)

\section{Histologische Untersuchung}

Für die Diagnosestellung einer septischen Arthritis ist die histologische Untersuchung von untergeordneter Bedeutung. Jedoch können wichtige Differenzialdiagnosen, wie eine Gichtarthropathie oder eine Chondrokalzinose, histologisch bewiesen werden und sollten deshalb durchgeführt werden. Die spezielle Untersuchung auf das Vorliegen einer Uratarthropathie oder Chondrokalzinose muss dabei im Auftrag der histologischen Untersuchung angegeben werden.

\section{Differenzialdiagnosen}

Von septischen Arthritiden sind alle Erkrankungen mit schmerzhaften, überwärmten, geröteten und geschwollenen Gelenken mit entsprechender Funktionseinschränkung zu differenzieren. Nicht in jedem Fall gelingt eine schnelle Differenzialdiagnose, was im weiteren diagnostischen und therapeutischen Vorgehen beachtet werden muss.

\section{Aktivierte Arthrose}

Durch stärkere Belastung oder spontan kann es in arthrotisch geschädigten Gelenken, vor allem im Hüft-, Knieund Sprunggelenk, im Sinne von Reparationsmechanismen zu mitunter ausgeprägten Entzündungsreaktionen kommen, typischerweise ist der Allgemeinzustand der Patienten gut, Fieber ist i.d. R. nicht vorhanden, die Therapie ist primär symptomatisch (Schmerzmittel, initiale Ruhigstellung des Gelenks). 


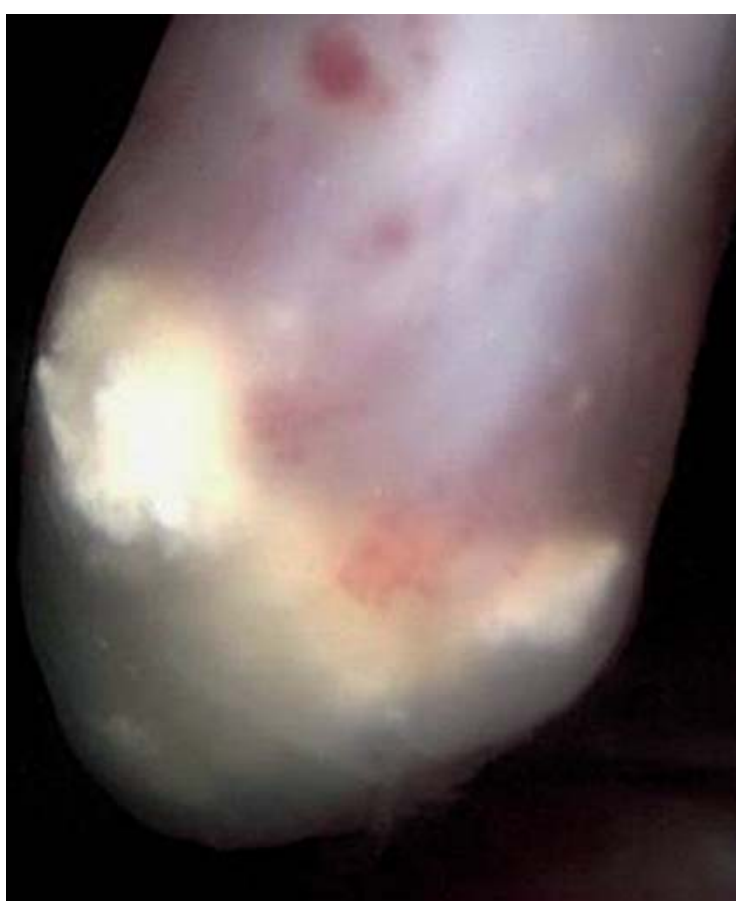

- Abb. 7 Synovialzotte mit histologisch gesicherten Urateinlagerungen (Gichtarthropathie des Kniegelenks).

\section{Gichtarthropathie}

Uratablagerungen ( $\bullet$ Abb. 7) können zu erheblichen Entzündungen von Gelenken führen. Neben dem Großzehengrundgelenk ist das Kniegelenk häufig betroffen. Der Allgemeinzustand kann beeinträchtigt sein, auch Fieber kann auftreten. Das CRP kann wie bei Infektionen sehr stark erhöht sein, das PCT ist typischerweise im Normbereich. Die Therapie ist konservativ mit nicht steroidalen Antiphlogistika (NSAR) und Urikostatika.

\section{Borreliose (Lyme-Arthritis)}

Wochen bis Monate nach einem Zeckenbiss können kulturnegative Arthritiden an verschiedenen Gelenken, meist dem Kniegelenk, auftreten. Die Anamnese bez. eines Zeckenbisses kann leer sein. Die Diagnose gelingt serologisch, die Therapie besteht in einer antibiotischen und symptomatischen Therapie.

\section{Reaktive Arthritiden (parainfektiöse Arthritiden)}

Tage bis wenige Monate nach einer gastroenterologischen oder urogenitalen Infektion können kulturnegative Arthritiden an einem oder mehreren Gelenken auftreten. Treten zusätzlich eine Urethritis und eine Konjunktivitis oder Iritis auf, spricht man von einem Reiter-Syndrom. Die Diagnose erfolgt serologisch, wobei eine Vielzahl von Erregern parainfektiöse Arthritiden verursachen können. Die Therapie ist abhängig vom Erreger und dem Krankheitsverlauf. Insbesondere Infektionen durch Chlamydia trachomatis müssen antibiotisch behandelt werden, da die Erkrankung die häufigste Ursache ungewollter Kinderlosigkeit in Deutschland darstellt. Eine gynäkologische Mitbehandlung ist obligat. Neben der Serologie führt der Direktnachweis bei der gynäkologischen Untersuchung durch eine Polymerasekettenreaktion (PCR) zur Diagnose.

\section{Erkrankungen des rheumatischen Formenkreises}

Bei einer Vielzahl von Erkrankungen des rheumatischen Formenkreises können Arthritiden und Arthralgien auftreten, einige wichtige seien im Folgenden genannt.

- rheumatoide Arthritis (RA): chronische, meist symmetrische Polyarthritis, typischerweise an kleinen Gelenken beginnend, Superinfektionen können auftreten ( $\triangleright$ Abb. 6)

- Kollagenosen (Lupus erythematodes, Sklerodermie, Mischkollagenose)

- akute Sarkoidose: klassische Trias aus Arthralgie, Erythema nodosum und bilateraler Lymphadenopathie im Thoraxröntgenbild

- Morbus Bechterew: meist asymmetrische Arthritis der Sakroiliakalgelenke und Wirbelgelenke, gehäuft bei jungen Männern zwischen dem 20. und 30. Lebensjahr beginnend, in >90\% HLA-B27-positiv

- Psoriasisarthritis: in etwa 10\% der Psoriasiserkrankungen kommt es zur begleitenden Arthritis, typischerweise an Händen, Füßen und Wirbelsäule

\section{Therapie}

\begin{abstract}
Merke
Die Behandlung der septischen Arthritis besteht aus den 3 Säulen operative, antibiotische und symptomatisch-funktionelle Therapie. Hierarchische Ziele der Therapie sind nach der Infektsanierung die Schmerzfreiheit, Stabilität und Mobilität des Gelenks [6]. „Die Gelenkinfektion ist ein dringlicher chirurgischer Notfall, gehört in stationäre Behandlung und erfordert die sofortige chirurgische Therapie. Die erfolgreiche Behandlung ist eine Funktion des Faktors Zeit." [6]

Aufgrund der erheblichen Konsequenzen der verzögerten Behandlung eines Gelenkinfekts ist bereits der Verdacht auf das Vorliegen einer septischen Arthritis eine Indikation zur operativen Therapie.
\end{abstract}

\section{Operative Therapie}

Prinzipiell kann die Primäroperation arthroskopisch und offen-chirurgisch erfolgen. Die Wahl des Verfahrens ist dabei abhängig vom klinischen Stadium bzw. der Zeitdauer der Infektion, vom betroffenen Gelenk und von der Erfahrung des Operateurs bez. der arthroskopischen Therapie des entsprechenden Gelenks. Bei Vorliegen eines Frühinfekts am Kniegelenk, Schultergelenk und Sprunggelenk ist die arthroskopische Operation im eigenen praktischen Vorgehen Methode der Wahl. Am Hüft- 


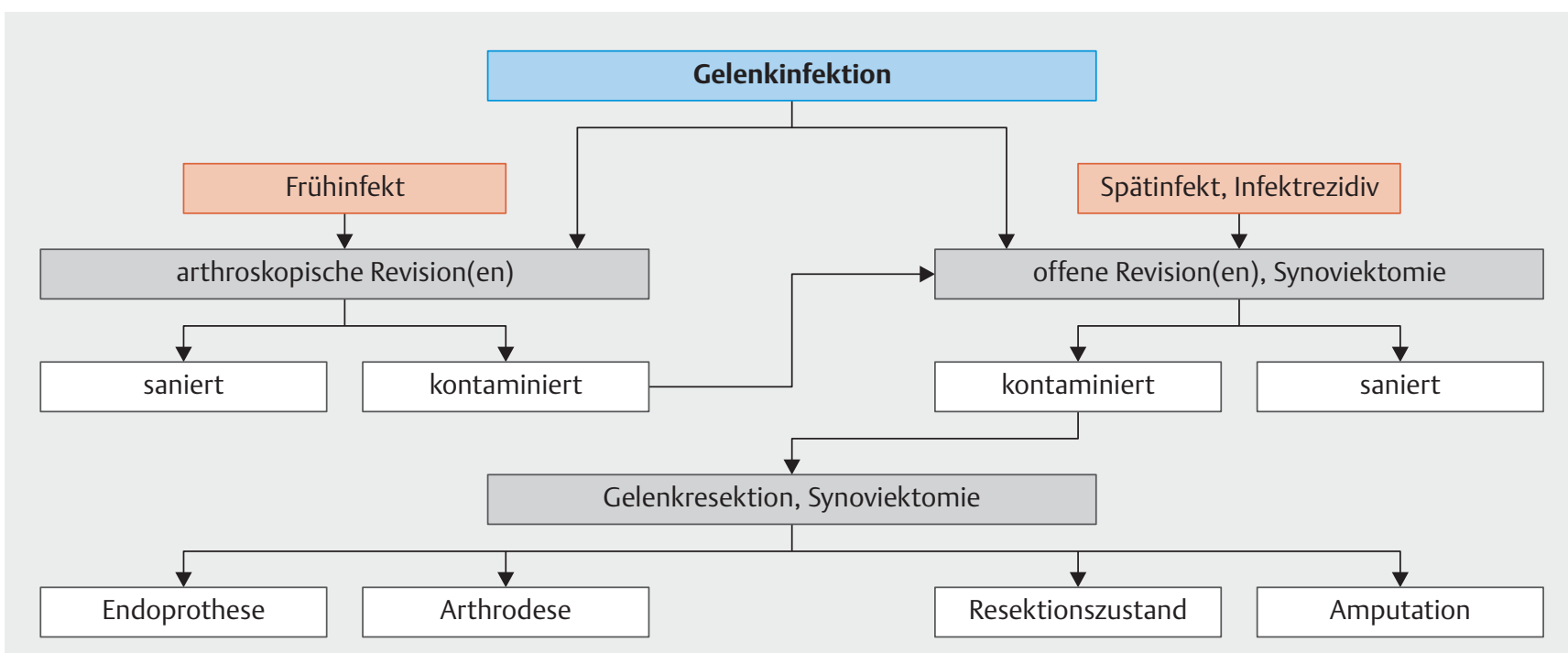

- Abb. 8 Algorithmus zur Behandlung von Gelenkinfektionen, nach Hofmann, 2004 [6].

gelenk, Ellenbogengelenk und Handgelenk ist bei entsprechender Erfahrung des Operateurs ebenfalls die primäre arthroskopische Behandlung, bei unzureichender Expertise die offen-chirurgische Therapie indiziert. Alle anderen Gelenkinfektionen werden primär offen-chirurgisch angegangen. Unabhängig vom chirurgischen Verfahren besteht die operative Behandlung in der Gewinnung repräsentativer mikrobiologischer Proben (mindestens 3 Proben aus dem Gelenkerguss und dem Synovialgewebe) sowie der ausgiebigen Spülung des Gelenks (mindestens 3 Liter). Auf den Zusatz antiseptischer und antibiotischer Substanzen wird aufgrund der möglichen zusätzlichen Schädigung des Gelenkknorpels und des fraglichen Nutzens verzichtet. Beim Frühinfekt erfolgt dabei in den Gächter-Stadien I und II keine ausgedehnte Synovektomie. Abschließend erfolgt die Einlage einer Drainage, wobei die Liegedauer so kurz wie möglich gehalten wird (maximal 2 Tage). Auf eine Saug-Spül-Drainage wird aufgrund mehrerer Nachteile (mangelnde Sterilität, hoher pflegerischer Aufwand, „Spülstraßenbildung“) und nicht gesicherten Nutzens generell verzichtet [1]. Auch wenn insbesondere in frühen Stadien der Infektion eine einmalige arthroskopische Operation zur Sanierung einer septischen Arthritis ausreichend sein kann [10], führen wir alle Patienten nach 3 bis 5 Tagen, aufgrund der erheblichen Konsequenzen einer unzureichenden Therapie, einer programmierten Lavage zu. Lässt sich in 3 arthroskopischen Operationen (wiederholte mikrobiologische Probeentnahmen) keine vollständige Infektsanierung erreichen, führen wir wie beim Spätinfekt oder den Gächter-Stadien III und IV eine vollständige offen-chirurgische oder kombinierte arthroskopisch-offene Synovektomie durch. Eine temporäre Ruhigstellung des Gelenks in einem Fixateur externe ist in nur wenigen Fällen (hochgradige Instabilität) erforderlich. Bei chronischen, sehr schwerwiegenden Infektionen mit Zerstörung des Gelenkknorpels und begleitender Osteitis kann eine radikale Gelenkresektion zur Infektsanierung erforderlich werden. Perspektivisch sind dann im besten Falle der endoprothetische Gelenkersatz oder eine Arthrodese ( $\bullet$ Abb. 5) bzw. Resektionsarthroplastik durchzuführen. In sehr seltenen Fällen kann bei Unsanierbarkeit der Infektion oder schwerer Sepsis eine Amputation der Extremität indiziert sein. Eine Ausnahme in der Therapie einer septischen Arthritis stellt die Gonokokkenarthritis dar, die ausschließlich einer antibiotischen Therapie bedarf. - Abb. 8 zeigt unseren Algorithmus zur Behandlung von Gelenkinfektionen [6].

\section{Antibiotische Therapie}

Die antibiotische Therapie ist essenzieller Bestandteil der Behandlung einer Gelenkinfektion. Diese sollte unmittelbar nach der Gewinnung repräsentativer mikrobiologischer Proben begonnen werden. Für 14 Tage empfehlen wir eine kombinierte intravenöse Therapie, primär kalkuliert durch ein Cephalosporin der 2. Generation und ein Chinolon, im Fall einer schweren Sepsis mit potenzieller Lebensgefahr durch ein Carbapenem und Linezolid. Die Therapie wird nach Erhalt des Antibiogramms ggf. angepasst, wobei wir eine weitere Kombinationstherapie mit möglichst bakterizid wirkenden Antibiotika bei hohen Gewebskonzentrationen in Weichteilen, Knochen und Gelenken und unterschiedlichem Wirkmechanismus empfehlen. Geeignete Substanzen sind neben Betalaktamantibiotika Chinolone, Fosfomycin, Rifampicin, Cotrimoxazol, Daptomycin und andere. Der intravenösen Therapie schließt sich eine weitere 2- bis 4-wöchige orale Antibiotikatherapie an. Grunderkrankungen der Patienten (Niereninsuffizienz, Allergien) sind ebenso wie mögliche Nebenwirkungen (antibiotikaassoziierte Kolitis) entsprechend zu beachten. 


\section{Symptomatisch-funktionelle Therapie}

Um neben einer Heilung der Infektion eine dauerhafte Schmerzfreiheit, Stabilität und Mobilität des Gelenks zu gewährleisten, sind neben der chirurgischen und antibiotischen Therapie weitere therapeutische Maßnahmen obligat.

\section{Schmerztherapie}

Akute Gelenkinfektionen können stark schmerzhaft sein und bedürfen neben einer Behandlung mit NSAR im Sinne des WHO-Stufenschemas zur Schmerztherapie oft einer additiven Therapie mit Opiaten. Auch nach Rückgang der akuten Schmerzsymptomatik ist eine adäquate Schmerztherapie zur Gewährleistung einer intensiven Physiotherapie erforderlich, um bestmögliche funktionelle Ergebnisse zu erzielen. Regionale Schmerzkatheter können eine sinnvolle Ergänzung zur konventionellen Schmerztherapie darstellen.

\section{Physiotherapie}

Die initiale funktionelle Behandlung der akuten septischen Arthritis besteht in einer Ruhigstellung, Kühlung und ggf. Hochlagerung des Gelenks. Nach Rückgang der akuten Schmerzsymptomatik und der akuten Entzündungsreaktion stehen dagegen intensive aktive und passive Bewegungsübungen des Gelenks und ein Muskelaufbau zur Stabilisierung des Gelenks ganz im Vordergrund. Dabei kommen regelhaft Bewegungsschienen zum Einsatz. Ist die untere Extremität betroffen, schließt sich eine Gangschulung, ggf. unter initialer Teilbelastung an. Ist die obere Extremität betroffen, können ergotherapeutische Maßnahmen indiziert sein.

Weitere therapeutische Maßnahmen: Durch die regelhafte Immobilisierung des Gelenks ist eine Thromboseprophylaxe, insbesondere bei Arthritis der unteren Extremität, zwingend erforderlich, diese sollte bis zum Wiedererreichen der Vollbelastung fortgeführt werden. Dekubitus- und Pneumonieprophylaxe müssen aus dem gleichen Grund beachtet werden. Darmregulierende Maßnahmen können bei antibiotikainduzierten oder opiatinduzierten Motilitätsstörungen erforderlich sein. Bei Vorliegen einer schweren Sepsis ist eine intenivmedizinische Behandlung obligat.

\section{Nachsorge, Komplikationen, Prognose und Prävention}

\section{Nachsorge}

- regelmäßige klinische und paraklinische Kontrollen über einen mehrwöchigen Zeitraum (Rezidivgefahr)

- Wiederholungseingriffe (second look) nach intraoperativem Befund und postoperativem Verlauf

- Anpassung der antibiotischen Therapie nach mikrobiologischen Befunden

- Physiotherapie, Ergotherapie und Rehabilitation
- ggf. Verordnung von Orthesen, Bandagen und Einlagen

- Planung rekonstruktiver chirurgischer Eingriffe bei schlechtem funktionellem Ergebnis nach Sanierung der Infektion, sofern konservative Therapien nicht ausreichend sind

\section{Merke}

Nach der Entlassung aus der stationären Behandlung führen wir alle Patienten mit einer Gelenkinfektion, unabhängig vom Versicherungsstatus, Kontrollen in unserer Spezialsprechstunde für septische und rekonstruktive Chirurgie zu, um Komplikationen frühzeitig erkennen und therapieren zu können.

\section{Komplikationen}

Die wichtigsten Komplikationen sind:

- akute chirurgische Komplikationen (Nachblutung, Gefäß-Nerven-Schäden, Sekundärinfektionen, Weichteildefekt, Thrombembolie)

- Medikamentenunverträglichkeit

- Sepsis bis zum Multiorganversagen und Exitus letalis

- hämatogene Sekundärinfektionen (Endoprotheseninfektionen, Endokarditis, Spondylodiszitis, septische Arthritiden anderer Gelenke)

- Rezidivinfektionen

- Chronifizierung der Infektion

- Zerstörung der Gelenkstrukturen (insbesondere Gelenkknorpel)

- Osteomyelitis der angrenzenden Knochen

- chronische Schmerzen im betroffenen Gelenk

- Instabilität des Gelenks

- Einschränkung der Mobilität des Gelenks bis zur Versteifung ( $\triangleright$ Abb. 5)

- Amputationen

\section{Prognose}

Bei frühzeitiger adäquater Behandlung einer septischen Arthritis und guter Compliance des Patienten ist die Prognose sowohl in Bezug auf das Überleben als auch die Funktion des Gelenks gut. Erfolgt der Beginn der Therapie jedoch verzögert oder fehlerhaft, liegen schwerwiegende Grunderkrankungen vor, ist die Virulenz der verursachenden Erreger hoch (superantigenproduzierende Staphylokokken, Pseudomonas aeruginosa) oder sind die Patienten sehr alt, kann die Mortalität infektiöser Arthritiden im Rahmen septischer Verläufe 20\% und mehr betragen [9]. Bei Beteiligung mehrerer Gelenke steigt die Mortalität auf bis zu 40\% [1]. Die Mortalität hat sich in den letzten 40 Jahren trotz großer Fortschritte in der Medizin nicht wesentlich verbessert. Wesentliche Funktionseinbußen des Gelenks werden, wiederum in Abhängigkeit von Behandlungsbeginn, Art der Behandlung, Alter und Grunderkrankungen, in 10 bis $73 \%$ gesehen [9]. 


\section{Merke}

Die frühzeitige adäquate Therapie der septischen Arthritis ist der wichtigste beeinflussbare Faktor zur Senkung der Sterblichkeit und zum Erhalt der Funktion des Gelenks.

\section{Prävention}

Aufgrund der schwerwiegenden Komplikationen einer septischen Arthritis bis hin zum Exitus letalis haben präventive Maßnahmen zur Verhinderung einer Infektion eine hohe Bedeutung.

- umgehend adäquate chirurgische Wundversorgung bei Verletzungen in Gelenknähe

- sicherer Ausschluss oder Nachweis einer primären Gelenkbeteiligung bei Verletzungen in Gelenknähe

- umgehende Behandlung von Infektionen in Gelenknähe (bakterielle Bursitiden)

- Vermeidung verschiebbarer gelenknaher Eingriffe und Operationen bei Vorliegen lokaler, regionaler und systemischer Infektionen

- Behandlung prädisponierender Faktoren für Gelenkinfektionen

- strenge Indikationsstellung von Gelenkpunktionen

- strenge Beachtung der Asepsis bei Gelenkpunktionen wie bei einer Operation

- frühzeitige Behandlung postoperativer Hämatome

- Antibiotikaprophylaxe vor Gelenkoperationen

- Sanierung anderer Infektionsherde

- Prävention von Folgeschäden (Physiotherapie)

\section{Interessenkonflikt}

Nein.

Über die Autoren
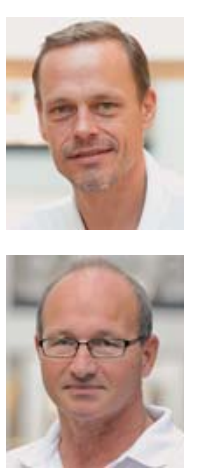

\section{Marc Hückstädt}

Dr. med., Oberarzt der Abteilung für septische und rekonstruktive Chirurgie, Klinik für Unfallund Wiederherstellungschirurgie, BG Klinikum Bergmannstrost Halle

\section{Gunter O. Hofmann}

Prof. Dr. Dr., Direktor der Klinik für Unfall- und Wiederherstellungschirurgie, BG Klinikum Bergmannstrost Halle
Korrespondenzadresse

Dr. med. Marc Hückstädt

Klinik für Unfall- und Wiederherstellungschirurgie BG Klinikum Bergmannstrost Halle

Merseburger Straße 165

06112 Halle

marc.hueckstaedt@bergmannstrost.de

\section{Literatur}

[1] Bonnaire F, Weber A. AWMF-Register Nr. 012/010 Klasse: S1. Bakterielle Gelenkinfektionen. Leitlinienkommission der Deutschen Gesellschaft für Unfallchirurgie e.V. (DGU) in Zusammenarbeit mit der Österreichischen Gesellschaft für Unfallchirurgie (ÖGU)

[2] Carpenter CR, Schuur JD, Everett WW. Evidence-based diagnostics: adult septic arthritis. Acad Emerg Med 2011; 18: 781-796

[3] Gächter A. Die Bedeutung der Arthroskopie beim Pyarthros Unfallheilkunde 1988; 200: 132-136

[4] Galloway JB, Hyrich KL, Mercer LK et al. Risk of septic arthritis in patients with rheumatoid arthritis and the effect of anti-TNF therapy: results from the British Society for Rheumatology Biologics Register. Ann Rheum Dis 2011; 70: 1810-1814

[5] Geirsson AJ, Statkevicius S, Víkingsson A. Septic arthritis in Iceland 1990-2002: increasing incidence due to iatrogenic infections. Ann Rheum Dis 2008; 67: 638-643

[6] Hofmann GO. Infektionen der Knochen und Gelenke. München, Jena: Urban \& Fischer; 2004

[7] Kuner EH, Thurck HU, von der Lippe I. Zur Diagnostik und Therapie der akuten Kniegelenkinfektion. Unfallchirurgie 1987; 13: $249-254$

[8] Simank HG, Wadi B, Bernd L. Gelenkempyeme. Orthopäde 2004; 33: 327-331

[9] Shirtliff ME, Mader JT. Acute septic arthritis. Clin Microbiol Rev 2002; 15: 527-544

[10] Stutz G, Gächter A. Diagnosis and stage-related therapy of joint infections. Unfallchirurg 2001; 104: 682-686

Bibliografie

DOI https://doi.org/10.1055/s-0043-102329

Online-publiziert 30.05.2017 | OP-JOURNAL 2017; 33: 118126 (c) Georg Thieme Verlag KG Stuttgart · New York ISSN 0178-1715 